Conclusions Pakistani infants were smaller in all measurements but had a tendency to greater central obesity (as indicated by subscapular skinfold thickness). Differences do not appear to differ markedly across generations. This suggests that differences may be genetically driven or are affected by epigenetic or persisting behaviour characteristics.

\section{P1-546 MALARIA INFECTION IN PREGNANCY AS A RISK FACTOR FOR LOW BIRTH WEIGHT IN THE DISTRICT OF BANGKA BELITUNG, INDONESIA, 2010}

doi:10.1136/jech.2011.142976h.33

II Winarta, ${ }^{1}$ Tafwid. ${ }^{1}$ Indonesia University, Depok, west Java, Indonesia; ${ }^{2}$ Gajah Mada University, Centre of Java, Indonesia

Background Malaria is a major health problem in Indonesia. The District of Bangka is one of 338 malaria endemic areas with a clinical malaria rate of $51.87 \%$ and positive malaria rate of $9.46 \%$ in 2008 . In malaria endemic areas pregnant mothers are the major group at risk for malaria infection. Malaria infection during pregnancy contributes to maternal morbidity and low birth weight (LBW) infants $(<2500 \mathrm{~g})$ caused either by preterm birth or intrauterine growth restriction (IUGR), factors that become the greatest risk factors for infant morbidity and mortality.

Methods This was a case-control study where the population are infants, namely LBW infants $(<2500 \mathrm{~g})$ as a case and normal birth weight infants $(2500-4000 \mathrm{~g})$ as a control within the period of 2008-2009. There were 174 subjects in total, comprising 58 cases and 116 controls. Data analysis used univariable, bivariable $\left(\chi^{2}\right)$ and multivariable (logistic regression) techniques.

Results There was a significant association between malaria infection in pregnant mothers and the incidence of LBW infants (crude $\mathrm{OR}=2.90 ; 95 \%$ CI 1.38 to 6.10 ), and after adjustment or controlled simultaneously for external variables, the influence of malaria infection remained (adjusted $\mathrm{OR}=2.97$; 95\% CI 1.37 to 6.39). Pregnant mothers with malaria infection had a risk 2.9 times greater for delivering LBW infants than normal birth weight infants. All external variables had no influence on malaria infection in pregnant mothers and the incidence of LBW infants.

Conclusion Pregnant mothers who delivered infants with LBW were more likely to be infected with malaria than pregnant mothers who delivered infants with normal birth weight.

\section{P1-547 ASSOCIATION OF DNA METHYLATION WITH METAL EXPOSURE AMONG HEALTHY KOREAN POPULATION}

doi:10.1136/jech.2011.142976h.34

H D Woo, D Paek, Y J Kim, Y Lee, J W Lee, H W Chung.* School of Public Health, Seoul national University, Seoul, Republic of Korea

Introduction DNA methylation is regarded as a well-defined epigenetic mechanism and involved in biological processes including ageing and cancer. The purpose of this study is to find whether DNA methylation is associated with lifestyle and environmental factors in healthy population residing in an industrialised zone in Korea.

Methods A total of 758 subjects (324 males and 434 females) were included in this study. Global DNA methylation level by lifestyle factors and environmental pollutants was measured in peripheral blood leukocyte.

Results Global DNA methylation levels (expressed as PMR values) of men were varied with age, while no change was seen in women. No association between lifestyle and biochemical factors (smoking, homocysteine, leptin, albumin and BMI) with global DNA methylation was observed. The relationship between metal such as mercury, cadmium and arsenic and DNA methylation was analysed Arsenic only had positive association with DNA methylation level in male group. Global DNA methylation pattern among family members was analysed to find familial aggregation. Significant associations of Mother with offspring, offspring with offspring were found. Correlation between sibling pairs was also increased in young age group, suggesting that DNA methylation patterns determined not only by genetic factors but environmental factors.

Conclusion DNA methylation levels of men were varied with age, while those of women were consistent throughout the age group. Positive association was shown in urinary arsenic only in adult male. DNA methylation level can be regulated by environmental stressor, but the association between each environmental factor and global DNA methylation was not strong.

\section{P1-548 RISK FACTORS FOR AN OUTBREAK OF CHIKUNGUNYA FEVER IN WEST BORNEO, INDONESIA, 2010}

doi:10.1136/jech.2011.142976h.35

F Y Sitepu, * A Suprayogi, D Pramono. Field Epidemiology Training Program (FETP) Gadjah Mada University, Yogyakarta, Indonesia

Background Chikungunya fever is a vector-borne disease with high morbidity rates, prolonged polyarthritis in some cases and it cause substantial socioeconomic impact. On 4 January 2010, an outbreak of Chikungunya fever was reported in West Borneo in Indonesia. The total number of cases was 65 with no fatalities. An analytical study was undertaken to determine the risk factors for the outbreak. Method This was an observational study with case control design. Cases were those with major clinical symptoms of Chikungunya fever, such as fever, arthalgia, myalgia, rash and headache. Controls were neighbours of cases who did not have clinical symptoms of the disease. The study used bivariate and multivariate analyses with $\chi^{2}$ and logistic regression. OR was used to determine risk factors. Some patient's blood was tested to confirm the diagnosis.

Results From the bivariate analyses, risk factors were farming in the forest ( $p$ value $<0.05, O R=10.14,95 \%$ CI 3.84 to 26.76); not using mosquito repellent devices ( $\mathrm{p}=0.001, \mathrm{OR}=4.70$, CI 1.75 to 12.60 ); not eradicating mosquito nests $(p=0.007, O R=3.18$, CI 1.33 to 7.61); and hanging clothes $(\mathrm{p}=0.015, \mathrm{OR}=2.814$, CI 1.206 to 6.566). The multivariate analyses showed that farming farming in the forest was an independent risk factor for infection (Exp.(B): 9.122, $p<0.05)$. Laboratory examination of blood samples confirmed that two out of four cases were positive for Chikungunya fever.

Conclusions Farming in the forest was a risk factor for infection. This was the basis for health promotion and prevention of future cases of Chikungunya fever in West Borneo.

\section{P1-549 THE ROLE OF PSYCHOSOCIAL FACTORS IN SLEEP DURATION IN ELDERS AND ITS EFFECT ON ALL-CAUSE MORTALITY: THE KRAKOW STUDY}

doi:10.1136/jech.2011.142976h.36

${ }^{1} \mathrm{~K}$ Zawisza, ${ }^{1} \mathrm{~B}$ Tobiasz-Adamczyk, ${ }^{2} \mathrm{~A}$ Galas, ${ }^{1} \mathrm{M}$ Brzyska. ${ }^{1}$ Department of Medical Sociology, Chair of Epidemiology and Preventive Medicine, Jagiellonian University Medical College, Krakow, Poland; ${ }^{2}$ 2Department of Epidemiology, Chair of Epidemiology and Preventive Medicine, Jagiellonian University Medical College, Krakow, Poland

Introduction Epidemiological studies performed in the last decades confirmed the significant role of sleep as an independent predictor of survival in older age.

Objective The aim of the study was to assess the role of the social position, self-reported functional status, self-rated health, and a 
feeling of life-weariness as risk factors of sleep duration, and to evaluate how the relationship between sleep duration and these psychosocial factors influences the risk of death.

Methods Baseline study using a structured questionnaire was performed in $1986 / 1987$ on a random sample $(n=2605)$ of older people aged 65 years and over, community-dwelling citizens of Krakow. Vital status was monitored over 22 years. The analysis was performed using logistic regression and Cox hazard models.

Results The mean observation period of the cohort was 11.5 years, for a total of 29951 person-years. 1341 deaths in women and 771 in men were recorded. Strong feeling of life-weariness was found as predictor of short sleep in both genders. Poor self-rated health in women and both low functional activity and low level of education in men was associated with a long sleep. Analysis showed increased risk of death in men who slept $<7 \mathrm{~h}$ and reported a strong feeling of life-weariness ( $\mathrm{HR}=2.2,95 \% \mathrm{CI} 1.2$ to 4.1), for women higher mortality was observed in those who reported long sleep and poor self-rated health ( $\mathrm{HR}=1.6,95 \%$ CI 1.2 to 2.2 ).

Conclusions Results suggest that sleeping patterns in elders depending on several psychosocial conditions influence the mortality patterns in older age.

\section{P1-550 SELF-RATED HEALTH AND MORTALITY IN A PROSPECTIVE CHINESE ELDERLY COHORT}

doi:10.1136/jech.2011.142976h.37

1J Zhou, ${ }^{1} \mathrm{~J}$ Johnston, ${ }^{2} \mathrm{~W}$ M Chan, ${ }^{2} \mathrm{Y}$ F Hui, ${ }^{2} \mathrm{P}$ S Ng, ${ }^{1} \mathrm{~T}$ H Lam, ${ }^{1} \mathrm{M}$ Schooling. ${ }^{*}$ ${ }^{1}$ Department of Community Medicine and School of Public Health, Li Ka Shing Faculty of Medicine, The University of Hong Kong, Hong Kong; ${ }^{2}$ Department of Health, The
Government of the Hong Kong Special Administrative Region, Hong Kong SAR, People's Republic of China, Hong Kong

Background Most previous studies of self-rated health (SRH) and mortality are in western populations and use global SRH. Conceptualisations of health and the cause-composition of mortality may be context specific; we examined the association of SRH with allcause and cause-specific mortality in a developed non-western population.

Methods We used multivariable Cox regression to examine, in 64189 Chinese people aged $65+$ years enrolled at 18 Elderly Health Centres from 1998 to 2001, the adjusted associations of age-comparative and self-comparative SRH with mortality and whether the associations varied by follow-up duration, sex, age or socio-economic position.

Results After a mean follow-up of 8.2 years, there were 12350 deaths. Worse age-comparative SRH was associated with higher mortality (HR 1.73, 95\% CI 1.62 to 1.85 ) than better SRH, adjusted for sex, age, education, housing, monthly expenditure, smoking, alcohol use, body mass index and physical activity. The associations varied by sex in the short term and weakened with age. Agecomparative SRH was more strongly associated with mortality from circulatory diseases (HR 1.93, 95\% CI 1.70 to 2.18) and respiratory diseases (HR 2.08, 95\% 1.79 to 2.42) than from cancer (HR 1.27, $95 \%$ CI 1.13 to 1.43 ). Worse self-comparative SRH was not associated with higher mortality.

Conclusion Age-comparative SRH is a good predictor of mortality in older people from a non-western setting but self-comparative $\mathrm{SRH}$ is not. However, the association was less marked than in western settings possibly because of contextually specific assessments of health or a different pattern of mortality. 